\title{
Scaling Laws for Dark Matter Halos in Late-Type and Dwarf Spheroidal Galaxies
}

\author{
John Kormendy ${ }^{1}$ and K. C. Freeman ${ }^{2}$ \\ ${ }^{1}$ Department of Astronomy, University of Texas at Austin, \\ 2515 Speedway, Mail Stop C1400, Austin, Texas 78712-1205, USA, \\ email: kormendy@astro.as .utexas.edu; \\ Max-Planck-Institut für Extraterrestrische Physik, \\ Giessenbachstrasse, D-85748 Garching-bei-München, Germany; \\ Universitäts-Sternwarte, Scheinerstrasse 1, D-81679 München, Germany; \\ ${ }^{2}$ Research School of Astronomy and Astrophysics, \\ Mount Stromlo Observatory, The Australian National University, \\ Cotter Road, Weston Creek, Canberra, ACT 2611, Australia, \\ email: Kenneth.Freeman@anu.edu.au
}

\begin{abstract}
Dark matter (DM) halos of Sc-Im galaxies satisfy structural scaling laws analogous to the fundamental plane relations for elliptical galaxies. Halos in less luminous galaxies have smaller core radii $r_{c}$, higher central densities $\rho_{\circ}$, and smaller central velocity dispersions $\sigma$. If dwarf spheroidal (dSph) and dwarf Magellanic irregular (dIm) galaxies lie on the extrapolations of these correlations, then we can estimate their baryon loss relative to that of $\mathrm{Sc}-\mathrm{Im}$ galaxies. We find that, if there had been no enhanced baryon loss relative to $\mathrm{Sc}-\mathrm{Im}$ galaxies, typical dSph and dIm galaxies would be brighter by $\Delta M_{B} \simeq-4.0 \mathrm{mag}$ and $\Delta M_{B} \simeq-3.5 \mathrm{mag}$, respectively. Instead, the galaxies lost or retained as gas (in dIm galaxies) baryons that could have formed stars. Also, dSph and dIm galaxies have DM halos that are more massive than we thought, with $\sigma \sim 30 \mathrm{~km} \mathrm{~s}^{-1}$ or circular-orbit rotation velocities $V_{\text {circ }} \sim 42 \mathrm{~km} \mathrm{~s}^{-1}$. Comparison of DM and visible matter parameter correlations confirms that, at $M_{V} \gtrsim-18$, dSph and dIm galaxies form a sequence of decreasing baryon-to-DM mass ratios in smaller dwarfs. We show explicitly that galaxy baryon content goes to (almost) zero at $V_{\text {circ }} \lesssim 42 \pm 4 \mathrm{~km} \mathrm{~s}^{-1}$, in agreement with $V_{\text {circ }}$ as found from our estimate of baryon depletion. Our results suggest that there may be a large population of DM halos that are dark and undiscovered. This helps to solve the problem that the initial fluctuation spectrum of cold dark matter predicts more dwarf galaxies than we observe.
\end{abstract}

\section{Introduction and Analysis Machinery}

This paper summarizes Kormendy \& Freeman (2014). That paper derives structural parameter correlations for DM halos in Sc-Im and dSph galaxies. We restrict ourselves to objects that contain only two main components, a baryonic disk or main body and a DM halo. For galaxies with well measured H I rotation curves $V(r)$, the derived DM parameters come from published maximum-disk decompositions of $V(r)$ into visible and dark components. The halo model used is the nonsingular isothermal. At absolute magnitude $M_{B} \gtrsim-14, V$ is comparable to the velocity dispersion; then rotation curve decomposition is impossible. For these dwarf spheroidal (dSph) and dwarf Magellanic irregular (dIm) galaxies, we derive central DM densities using the Jeans equation.

We compare DM parameters with visible matter parameter correlations from Kormendy \& Bender (2012). They show that the parameter correlations of Sph galaxies are continuous with the disks (but not bulges) of S0 galaxies. In essence, Sph galaxies are bulgeless S0s. Moreover, Sph and $\mathrm{S}+\mathrm{Im}$ galaxies have similar structure at each $M_{V}$. Effective brightnesses decrease dramatically at $M_{V}>-18$; this is used in Figure 3 here. 


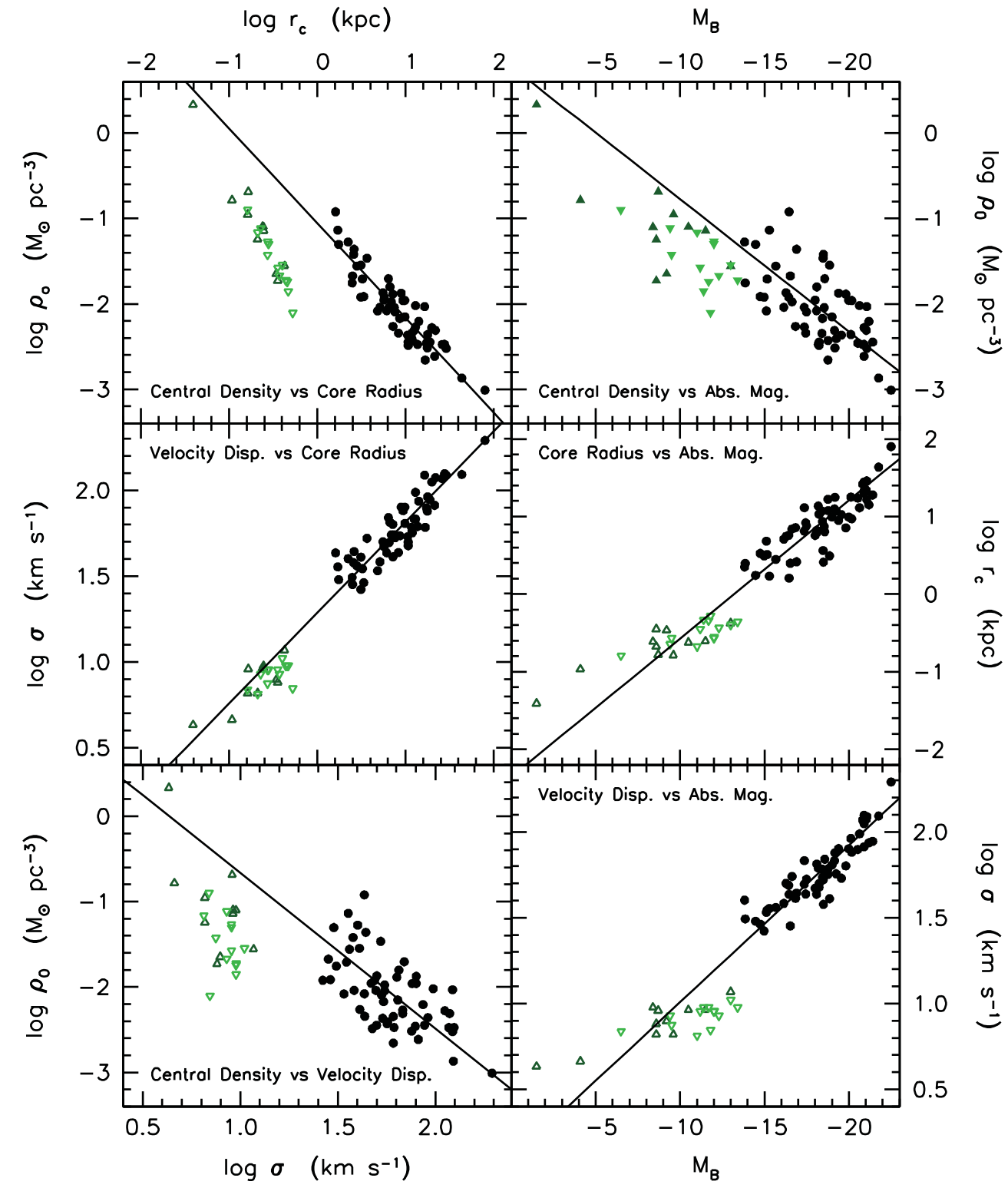

Figure 1. Dark matter parameter correlations for $\mathrm{Sc}-\mathrm{Im}$ galaxies as derived from rotation curve decompositions using the nonsingular isothermal as DM model (black points and black line = symmetric least-squares fit). Also added are dSph galaxies (green triangles) and dIm galaxies (upside-down green triangles). For dSph and dIm galaxies, $\rho_{\circ}$ is a meaure of the DM and therefore is plotted with filled symbols in the top-right panel. But $r_{c}$ and $\sigma$ are visible-matter parameters and so are plotted with open symbols in the other panels. From Kormendy \& Freeman (2014).

\section{DM Structural Parameter Correlations}

Figures 1 and 2 show the main observational result of Kormendy \& Freeman (2014): $\mathrm{DM}$ halos of $\mathrm{Sc}-\mathrm{Im}$ galaxies satisfy well defined scaling laws. Halos in less luminous galaxies have smaller core radii, higher central densities, and smaller velocity dispersions. This confirms previous analyses of smaller samples (Kormendy 1988, 1990; Kormendy \& Freeman 2004). Scaling laws provide new constraints on galaxy formation. For example: 


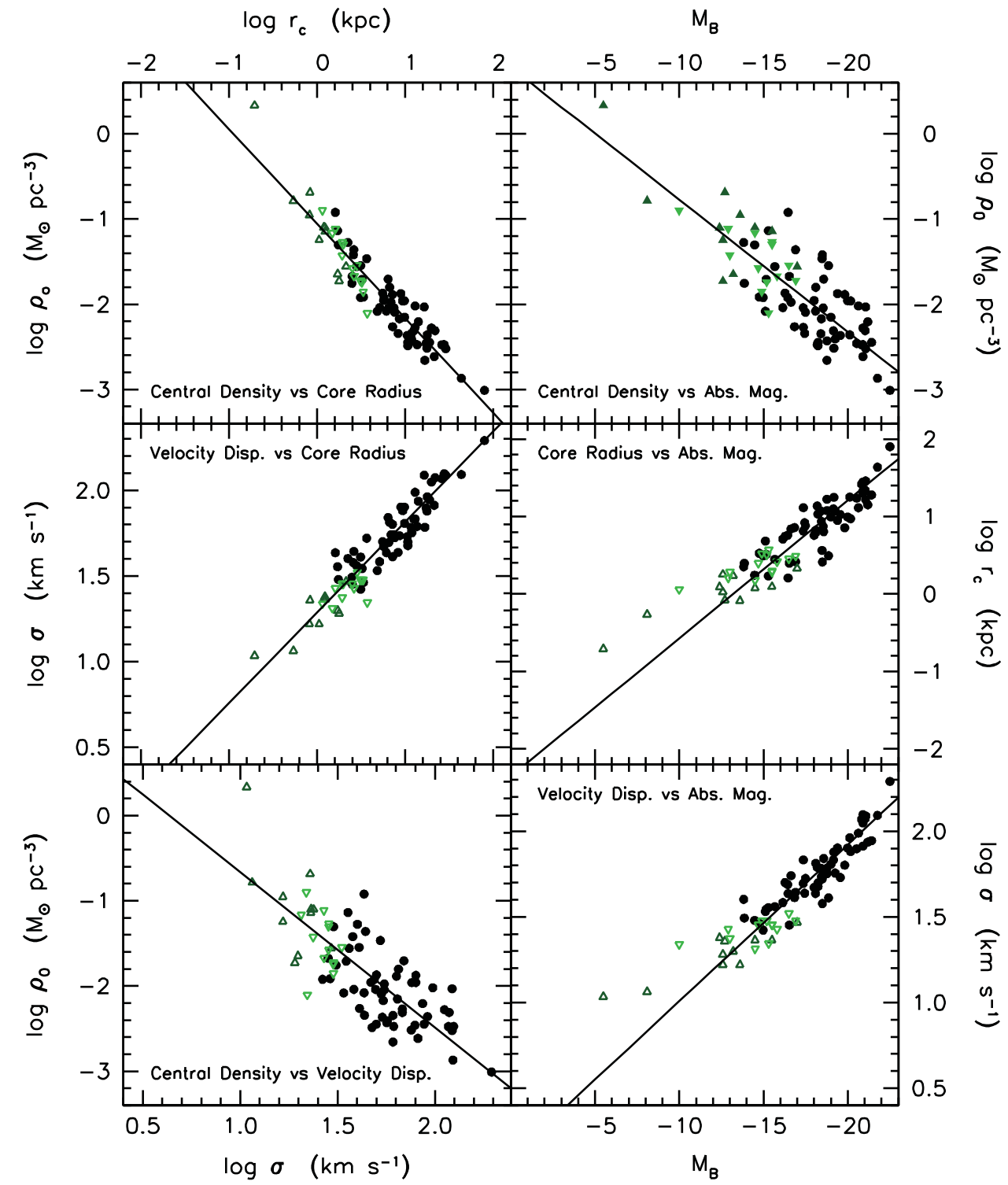

Figure 2. DM correlations for $\mathrm{Sc}-\mathrm{Im}$, dSph, and dIm galaxies (Figure 1) but with dSph and dIm galaxies shifted in $M_{B}, \log r_{c}$, and $\log \sigma$ (but not $\log \rho_{\circ}$ ) to move them onto the scaling laws for the $\mathrm{Sc}-\mathrm{Im}$ galaxies. The goal is to estimate (1) the baryon loss from dwarf galaxies relative to that from $\mathrm{Sc}-\mathrm{Im}$ galaxies and (2) the difference between the baryon and DM values of $\log r_{c}$ and $\log \sigma$. The shifts for dSph and dIm galaxies are, respectively, $M_{B} \rightarrow M_{B}-4.0$ and $M_{B} \rightarrow M_{B}-3.5, \log r_{c} \rightarrow \log r_{c}+0.70$ and $\log r_{c} \rightarrow \log r_{c}+0.85$, and $\log \sigma \rightarrow \log \sigma+0.40$ and $\log \sigma \rightarrow \log \sigma+0.50$. Note the interpretation of the shifts: $\Delta \log r_{c}$ gives us the ratio of DM core radius to visible matter core radius, and $\Delta \log \sigma$ gives us the ratio of DM velocity dispersion to visible matter velocity dispersion. From Kormendy \& Freeman (2014).

Halo density depends on collapse redshift $z_{\text {coll }}$ as $\rho_{\circ} \propto\left(1+z_{\text {coll }}\right)^{3}$. Thus $\rho_{\circ}$ increases toward lower luminosities because fainter galaxies collapsed earlier. The DM correlations imply that dwarf galaxies formed at least $\Delta z_{\text {coll }} \simeq 7$ earlier than giant spirals. Correction for baryonic DM compression would make the "pristine" $\rho_{\circ}$ smaller for giant galaxies and would increase $\Delta z_{\text {coll }}$. 


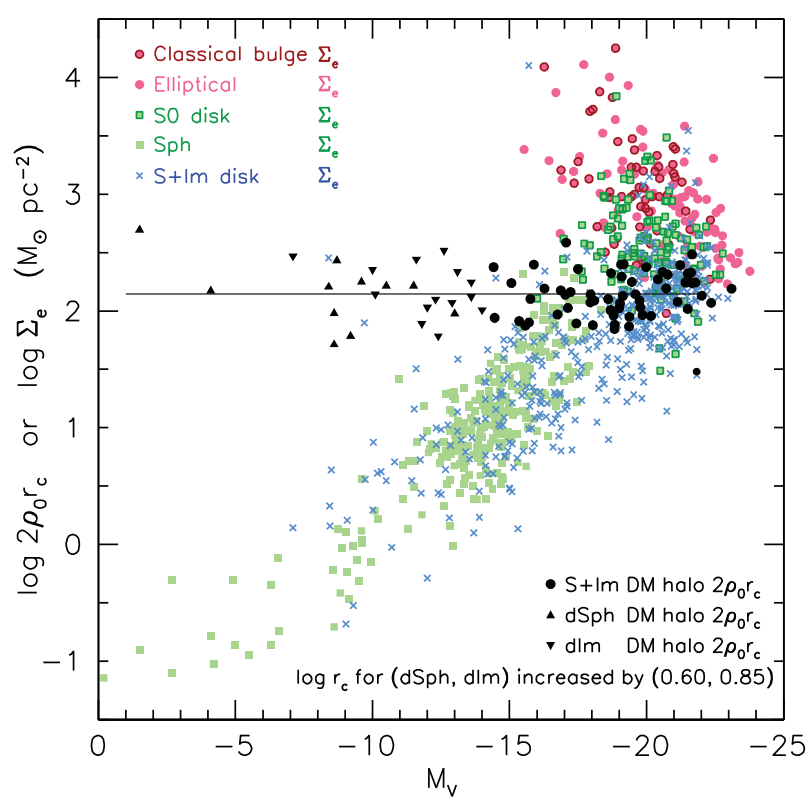

Figure 3. Comparison of DM halo parameters from Fig. 2 with visible matter galaxy parameters from Kormendy \& Bender (2012). Central projected densities are plotted for DM halos; effective surface densities $\Sigma_{e}=\Sigma\left(r_{e}\right)$ are shown for visible components. Here $r_{e}$ is the radius that encloses half of the light of the component. To convert surface brightnesses to stellar surface densities, we assume mass-to-light ratios $M / L_{V}=8$ for ellipticals, 5 for bulges and S0 disks, and 2 for spiral galaxy disks, Im galaxies, and Sph galaxies. From Kormendy \& Freeman (2014).

In Figure 3, smaller dwarfs have smaller stellar-to-DM ratios; these are probably associated with baryon loss. The correlations in Figure 1 provide a way to estimate this baryon loss and the properties of dwarf galaxy halos. The Jeans equation tells us the DM central density $\rho_{\circ}$ but not its core radius $r_{c}$ or velocity dispersion $\sigma$. So the top-right panel in Figure 1 correctly shows an offset of dSph+dIm galaxies from the extrapolation of the fitted $\rho_{\circ}-M_{B}$ correlation. But the smallest galaxies with rotation curve decompositions have DM densities similar to those of the biggest dwarfs with Jeans equation estimates. We assume that $d S p h+d I m$ galaxies would lie on the extrapolation of the $\log \rho_{\circ}-M_{B}$ correlation for bright galaxies except for the effects of their enhanced baryon loss. The top-right panel of Figure 1 then tells us the $\Delta M_{B}$ shifts that bring dSph and dIm galaxies onto the fitted relation. The top-left and bottom-left panels tell us the shifts in $\log r_{c}$ and $\log \sigma$ that bring $\mathrm{dSph}+\mathrm{dIm}$ galaxies onto those relations. These shifts are applied in Figure 2. In all panels, dSph and dIm galaxy halos lie on the correlations for more massive galaxies. The $\Delta \log \sigma$ shifts imply that almost-dark dwarfs are more massive than we thought. Their typical halo has $\sigma \sim 30 \mathrm{~km} \mathrm{~s}^{-1}$. This corresponds to $V_{\text {circ }} \sim 42 \mathrm{~km} \mathrm{~s}^{-1}$, in remarkably good agreement with the value of $V_{\text {circ }}$ where galaxies get $\operatorname{dim}$ in Figure 4 .

\section{Comparison of Scaling Relations for Visible and Dark Matter}

Figure 3 compares the central projected densities of DM halos with effective projected densities of stars. $\mathrm{S}+\mathrm{Im}+\mathrm{Sph}$ galaxies with $M_{V} \approx-18$ form a sequence of decreasing baryon-to-DM density ratios at decreasing $L_{V}$. We suggest that they form a sequence of decreasing baryon retention (vs. supernova-driven winds: Dekel \& Silk 1986) or decreasing baryon capture (after cosmic reionization) in smaller galaxies. For galaxies with present 


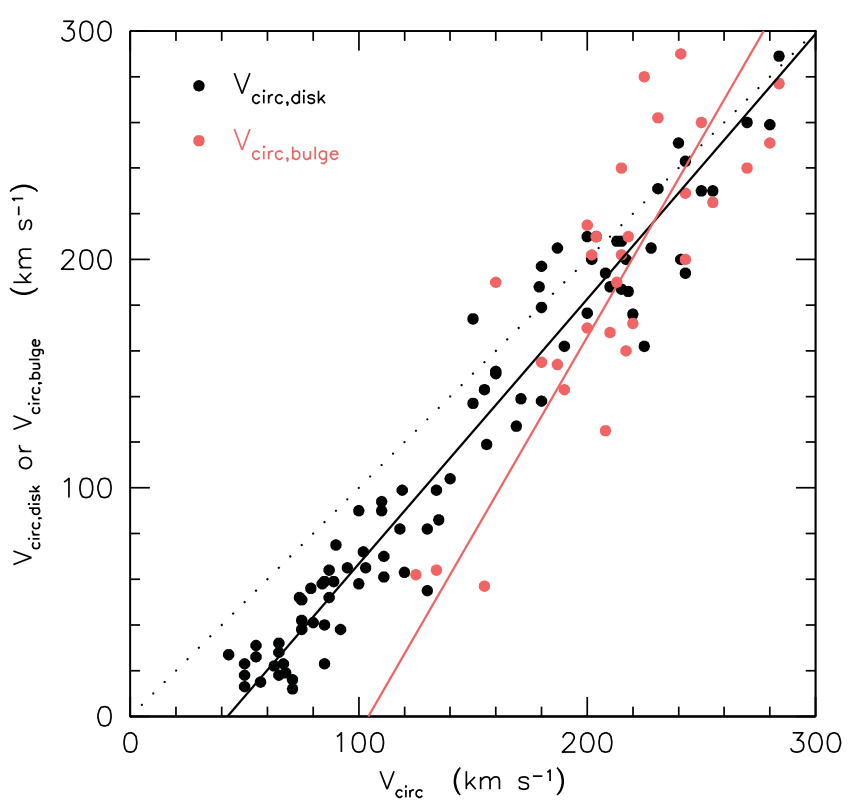

Figure 4. Maximum rotation velocity of the bulge $V_{\text {circ,bulge }}$ (red points) and disk $V_{\text {circ,disk }}$ (black points) given in bulge-disk-halo decompositions of galaxy rotation curves whose outer, DM test particle rotation velocities are $V_{\text {circ }}$. The dotted line indicates $V_{\text {circ,bulge }}=V_{\text {circ,disk }}=V_{\text {circ }}$. Every red point has a corresponding black point, but many galaxies are bulgeless, and then only a disk was included in the decomposition. This figure shows that the "rotation curve conspiracy", $V_{\text {circ,bulge }} \simeq V_{\text {circ,disk }} \simeq V_{\text {circ }}$ for the halo (Bahcall \& Casertano 1985; van Albada \& Sancisi 1986; Sancisi \& van Albada 1987), happens mostly for galaxies with $V_{\text {circ }} \sim 200 \mathrm{~km} \mathrm{~s}^{-1}$. The lines are least-squares fits with each variable symmetrized around $200 \mathrm{~km} \mathrm{~s}^{-1}$. The correlation for bulges is steeper than the one for disks; bulges disappear at $V_{\text {circ }} \sim 104 \pm 16 \mathrm{~km} \mathrm{~s}^{-1}$. Disks disappear robustly at $V_{\text {circ }}=42 \pm 4 \mathrm{~km} \mathrm{~s}^{-1}$. From Kormendy \& Freeman (2014).

$M_{V} \sim-10 \pm 3$, the baryon depletion is estimated by $\Delta M_{B}$ in the caption of Figure 2 . If the stellar $M / L_{V}$ is similar in dwarfs and $\mathrm{Sc}$-Im systems, then $\Delta M_{B}$ measures the mass of stars in dwarfs relative to the mass of stars in Sc-Im galaxies with halos of similar $\rho_{\circ}$. This agrees with their mass-to-light ratios, $M / L_{V} \sim 10^{2}$. These dwarfs are almost dark, probably because they lost more baryons than the (also substantial) loss from $\mathrm{Sc}-\mathrm{Im}$ galaxies. In dIm galaxies, some baryons are still in cold gas, but this effect is fairly small.

Also in Figure 3, projected (not volume!) DM density $\Sigma_{D M \circ}$ is essentially independent of galaxy luminosity $L_{V}$. This is a well known result (Kormendy \& Freeman 2004; Spano et al. 2008; Gentile et al. 2009; Donato et al. 2009; Plana et al. 2010). It implies a Faber-Jackson (1976) relation of the form DM mass $M_{D M} \propto \sigma^{4}$.

Finally, in Figure 3, bulges and elliptical galaxies have $\Sigma_{e}>\Sigma_{D M \circ}$, more so at lower $L_{V}$. Kormendy et al. (2009) and Kormendy \& Bender (2012) suggest that they form a sequence of increasing dissipation in the formation of smaller galaxies.

Perhaps the most remarkable result in Kormendy \& Freeman (2014) is shown in Fig. 4. It shows that rotation-curve decompositions reveal a robust, linear correlation between the maximum rotation velocity $V_{\text {circ,disk }}$ of baryonic disks and the outer circular velocity $V_{\text {circ }}$ of test particles in their DM halos. It explicitly shows that $V_{\text {circ,disk }} \rightarrow 0 \mathrm{~km} \mathrm{~s}^{-1}$ at $V_{\text {circ }}>0 \mathrm{~km} \mathrm{~s}^{-1}$. In fact, baryons become unimportant at $V_{\text {circ }}=42 \pm 4 \mathrm{~km} \mathrm{~s}^{-1}$. This $V_{\text {circ,disk }}=0$ intercept agrees very well with the typical halo $\sigma$ that we deduced in Section 2. Smaller galaxies are dim or dark. For example, the two extremely faint dSph galaxies in our sample, Segue 1 and Coma, have $V_{\text {circ }}$ values of about $16 \mathrm{~km} \mathrm{~s}^{-1}$. 


\section{Does There Exist a Large Population of Dark Galaxies?}

From the above results, we conclude that the range of visible matter content of $\sigma \sim 30$ $\mathrm{km} \mathrm{s}^{-1} \mathrm{DM}$ halos is large. The ones with the most baryons rotate enough to allow rotation curve decomposition. The ones with the fewest baryons are barely discoverable. None of these galaxies "know" that they must retain $\sim 1 \%$ of their baryons to be discoverable by us almost 14 billion years after they formed. Moreover, as luminosity decreases toward barely discoverable galaxies, these dwarfs become much more numerous as well as much more nearly dominated by DM. And baryon depletion processes should be more efficient in smaller galaxies. All this suggests that there may exist a large population of objects that are even darker - too dark to be discovered by current techniques. This would help to solve the problem that such objects are predicted by cold DM theory but not seen in Local-Group-like environments (Moore et al. 1999; Klypin et al. 1999).

\section{Acknowledgments}

JK thanks the Directors and staff of Mt. Stromlo Observatory (Australia), of the Observatory of the Ludwig-Maximilians-University (Munich, Germany), and of the Max-Planck-Institute for Extraterrestrial Physics (Garching-by-Munich, Germany) for hospitality and support during many visits over many years when this work was done. We made extensive use of NASA's Astrophysics Data System bibliographic services and of the NASA/IPAC Extragalactic Database (NED). NED is operated by the Jet Propulsion Laboratory and Cal Tech under contract with NASA. And we used the HyperLeda electronic database at http://leda.univ-lyon1.fr (Paturel et al. 2003). JK's work was supported by NSF grants AST-9219221 and AST-0607490, by the Alexander von Humboldt-Stiftung (Germany), and by Sonderforschungsbereich 375 of the German Science Foundation. The visits to Mt. Stromlo were made possible by the long-term support provided to JK by the Curtis T. Vaughan, Jr. Centennial Chair in Astronomy.

\section{References}

Bahcall, J. N., \& Casertano, S. 1985, ApJ, 293, L7

Dekel, A., \& Silk, J. 1986, ApJ, 303, 39

Donato, F., Gentile, G., Salucci, P., et al. 2009, MNRAS, 397, 1169

Faber, S. M., \& Jackson, R. E. 1976, ApJ, 204, 668

Gentile, G., Famaey, B., Zhao, H., \& Salucci, P. 2009, Nature, 461, 627

Klypin, A., Kravtsov, A. V., Valenzuela, O., \& Prada, F. 1999, ApJ, 522, 82

Kormendy, J. 1988, in Guo Shoujing Summer School of Astrophysics; Origin, Structure and Evolution of Galaxies, ed. Fang Li Zhi (Singapore: World Scientific), 252

Kormendy, J. 1990, in The Edwin Hubble Centennial Symposium: Evolution of the Universe of Galaxies, ed. R. G. Kron (San Francisco: ASP), 33

Kormendy, J., \& Bender, R. 2012, ApJS, 198, 2

Kormendy, J., Fisher, D. B., Cornell, M. E., \& Bender, R. 2009, ApJS, 182, 216

Kormendy, J., \& Freeman, K. C. 2004, in IAU Symposium 220, Dark Matter in Galaxies, ed. S. D. Ryder, D. J. Pisano, M. A. Walker, \& K. C. Freeman (San Francisco: ASP), 377

Kormendy, J., \& Freeman, K. C. 2014, ApJ, submitted (arXiv:1411.2170)

Moore, B., Ghigna, S., Governato, F., et al. 1999, ApJ, 524, L19

Paturel, G., Petit, C., Prugniel, P., et al. 2003, A\& A, 412, 45

Plana, H., Amram, P., Mendes de Oliveira, C., \& Balkowski, C. 2010, AJ, 139, 1

Sancisi, R., \& van Albada, T. S. 1987, in IAU Symposium 117, Dark Matter in the Universe, ed. J. Kormendy \& G. R. Knapp (Dordrecht: Reidel), 67

Spano, M., Marcelin, M., Amram, P., et al. 2008, MNRAS, 383, 297

van Albada, T. S., \& Sancisi, R. 1986, Phil. Trans. R. Soc. London A, 320, 447 